\title{
Electronic Properties and Complexity of Al-Based Complex Metallic Alloys
}

\author{
J.-M. Dubois ${ }^{a *}$ AND E. BELIN-FERRÉ ${ }^{b}$ \\ ${ }^{a}$ Institut Jean Lamour (UMR 7198 CNRS Université de Lorraine), Parc de Saurupt, CS 50840, 54011 Nancy, France \\ ${ }^{b}$ Laboratoire de Chimie-Physique, Matière et Rayonnement (UMR 7614 CNRS UPMC) \\ 11 rue Pierre et Marie Curie, 75005 Paris, France \\ As a consequence of their structural complexity, the electron transport properties of Al-based complex metallic \\ alloys scale as simple power laws of the number of atoms in the primitive unit cell. Furthermore, presence in energy \\ space of localised, $d$-like states below the Fermi level is systematically observed, even in the absence of any transition \\ metal constituting element. The relative intensity of this contribution to the total density of states correlates with \\ the structural complexity of the compound. Thus, complexity plays a key role, via Hume-Rothery, hybridization \\ effects and most probably hopping, in the selection, formation and stability of Al-based complex metallic alloys. \\ The results are interpreted in terms of self-organized criticality. In order to promote discussion about the essence \\ of the quasicrystalline state ("why are the atoms where they are?"), a speculative model is suggested.
}

DOI: 10.12693 /APhysPolA.126.453

PACS: 61.82.Bg; 61.44.Br; 71.55.Ak; 71.23.Ft; 72.15.-v

\section{Introduction}

In spite of years of effort dedicated to complex metallic alloys (CMAs) [1], the reason why quasicrystals form in a metallic alloy system of appropriate stoichiometry has so far found no satisfactory answer. Interaction between the Fermi surface and the Jones zone (Fs-Jz for short) was pointed out to explain the stability of the lattice owing to an interference condition [2] that is satisfied when the wavelength of nearly free electrons matches the distances formed between close packed atomic planes. Yet, this condition is obeyed as well in crystalline compounds of nearby composition in the phase diagram and therefore it does not allow to understand why nature allows quasicrystals to grow instead of more and more complex crystals.

Furthermore, chemical bonding between species contributes as well to stabilize the lattice structure since it participates in the formation of the pseudogap [3]. A systematic investigation over the years, using soft X-ray emission spectroscopy (SXES for short) has led to a quantitative analysis of the partial densities of $3 p$ and $3 s, d$ states at the aluminium edge and $3 d$ states at the transition metal edge [4]. Hybridization between $3 p$ and $d$ states is manifest. The question then arises to understand to which extent $d$-states present within the energy range that overlaps the Fermi level may contribute as well to the stability of the lattice. If the answer is positive, how and to which extent does the symmetry of $d$-states enter the selection mechanism of a specific lattice and determine its overall symmetry?

The paper is organized in an attempt to bring preliminary answers to those questions and trigger further theoretical research.

\footnotetext{
*corresponding author; e-mail: jean-marie.dubois@univ-lorraine.fr
}

\section{Samples and complexity of CMAs}

Several samples, all based on aluminium or magnesium, were used in the present study. The way they were grown from raw constituents is described elsewhere [5]. Symbols that refer to the samples used in the present study (except for $\mathrm{Al}-\mathrm{Mg}$ and $\mathrm{Al}-\mathrm{Mg}-\mathrm{Zn}$ alloys) are given in the inset of Fig. 1. They are referred to using standard notations from literature $[6,7]$.

It is difficult to rationalize what we call "complexity" of a CMA compound. In this article, we rely on a very straightforward method and define complexity using one single number called hereafter the complexity index

$$
\beta_{\mathrm{C}}=\ln N_{\text {u.c. }} \text {, }
$$

where $N_{\text {u.c. }}$ is the number of atoms found in the primitive unit cell of the compound without distinguishing chemical species. Accordingly, $\beta_{\mathrm{C}}$ is very close to the Shannon entropy requested to build up a single constituent crystal of identical lattice. With such a crude definition of complexity, it is clear that all details introduced by the specific chemistry of each alloy will be smeared out.

The size of the unit cell of a quasicrystal is said infinite. Yet, in order to avoid handling infinities, we may notice that the size of a sample is never infinite. Assuming a quantity of $1 \mathrm{at}$. g, or $6.022 \times 10^{23}$ atoms, in the volume of a single grain sample, one has $\beta_{\mathrm{C}}$ of the order of 55 , which is far from being infinite. Alternatively, $\beta_{\mathrm{C}}$ depends on the grain size in multi-grained quasiperiodic materials.

The experimental data we will deal with in the following sections are conductivity measurements [8-11] and SXES analysis of the partial densities of states (DOS for short) in $\mathrm{Al}-\mathrm{Mg}(-\mathrm{Zn})$ specimens [12]. Using the SXES technique, we will be able to separate the $3 s, d$ and $3 p$ contributions to the total DOS when measuring the emission spectra at the edges of $\mathrm{Al}$ and $\mathrm{Mg}$, respectively. 


\section{Electron transport and self-organised criticality}

The $4 \mathrm{~K}$ conductivity [8-11] shows a dramatic decay over few orders of magnitude, from high conductance in metallic fcc aluminium to very low conductivity in icosahedral monograins. When presented as a $\ln -\ln$ plot (Fig. 1), the dependence of the conductivity at $4 \mathrm{~K}$ as a function of the complexity index reduces to a straight line, with slope very close to -1 , on which all periodic CMAs fall, plus data points located far apart, which represent the non-periodic samples used in this study.

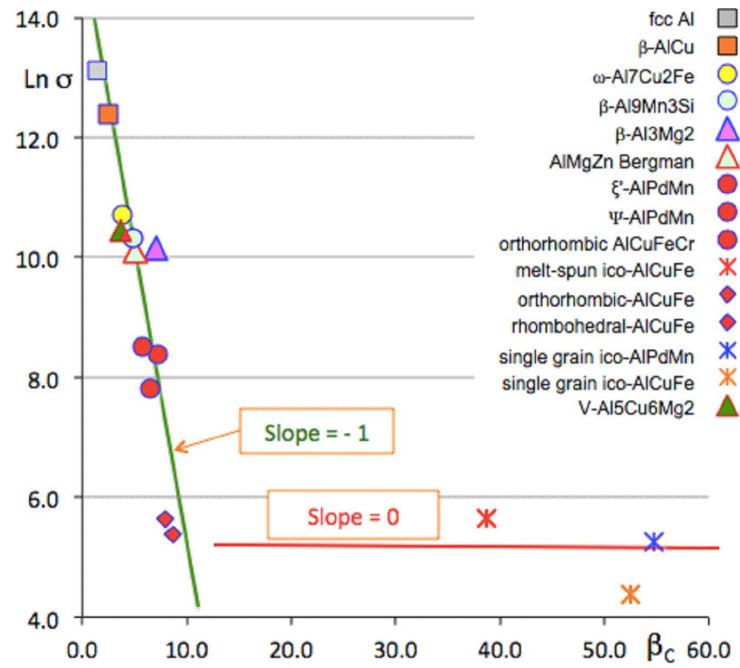

Fig. 1. Electronic conductivity $\sigma$ at $4 \mathrm{~K}$ of a number of Al-based CMAs, including quasicrystals and large unit cell approximants, versus the complexity index and presented as a $\ln (\sigma)$ vs. $\beta_{\mathrm{C}}=\ln \left(N_{\text {u.c. }}\right)$ plot. The two lines drawn in the figure are with a slope of -1 (in green) and 0 (in red), to guide the eye.

Hence, electron transport depends on lattice complexity index according to a very simple power law

$$
\sigma \propto \beta_{\mathrm{C}}^{-1}
$$

except for the most complex CMAs, which fall aside the plot, at the highest values of $\beta_{\mathrm{C}}$. This of course raises the question to know whether the present definition of the lattice complexity is optimum, but we shall leave this question aside since it will not affect our conclusions.

Many phenomena in nature show the same type of power law dependence. Such phenomena were studied in detail by Bak [13] who concentrated more specifically on the model given by a sand pile that reaches its equilibrium shape by producing avalanches of varying size on its slopes. He showed that the number of sand grains contained in an avalanche of a certain size is inversely proportional to its frequency of occurrence. The ensemble of phenomena ruled by a relationship like Eq. (2) was coined self-organised criticality (SOC for short) [14].

Propagation of electrons in a CMA can be approximated as an avalanche only on a macroscopic level. Yet, the shape of the plot presented in Fig. 1 clearly indicates that electron localisation is far more important in quasicrystals than in any periodic CMA known so far, a conclusion that was reached already long ago [15]. It is also known that electronic conductivity is a function of the DOS at the Fermi energy $E_{\mathrm{F}}$, the type of function being representative of the transport mechanism. We expect simple proportionality in the ballistic, or Einstein type of conduction whereas hopping (which is a thermally activated process) leads to a quadratic dependence on the DOS at $E_{\mathrm{F}}[16]$.

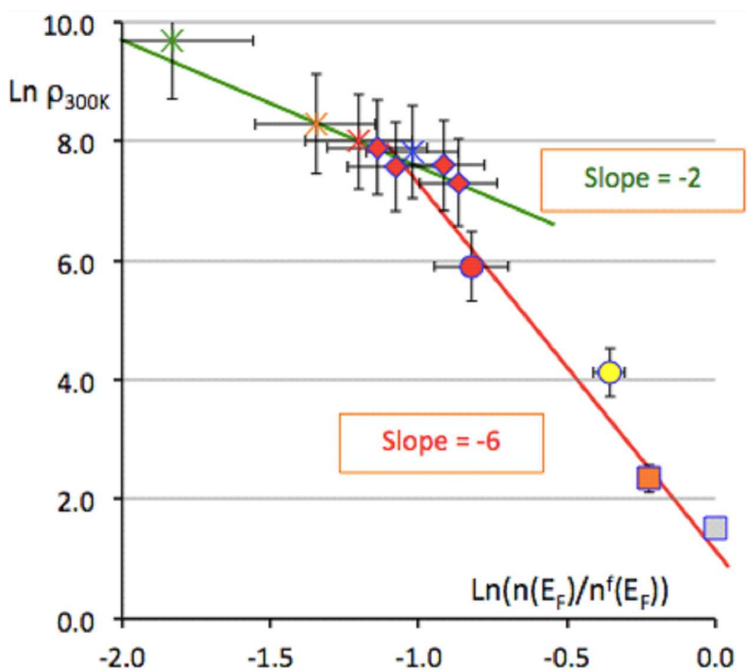

Fig. 2. $\ln \left(\rho_{300 \mathrm{~K}}\right)$ vs. $\ln (r)$ plot of the room temperature resistivity $\rho_{300 \mathrm{~K}}$ of several samples versus the ratio $r$ between the Al $3 p$ DOS at the Fermi energy obtained by SXES in the specimen and in a fcc $\mathrm{Al}$ reference sample. The meaning of the symbols is as defined in the inset of Fig. 1. Large unit cell CMAs collect in the left upper part of the figure whereas smaller unit cells are found in the lower right part. The two lines, with slopes -2 and -6 , respectively, guide the eye.

Figure 2 presents the logarithm of the room temperature resistivity $\rho_{300 \mathrm{~K}}$ of the same specimens as a function of the logarithm of the ratio $r=n\left(E_{\mathrm{F}}\right) / n^{\mathrm{f}}\left(E_{\mathrm{F}}\right)$ between $\mathrm{Al} 3 p$ DOSs measured in the sample of interest and in pure aluminium taken as a typical free electron system. According to Mizutani [16], a plot of $\ln (\rho)$ versus $\ln (r)$ is expected to be represented by a straight line with slope equal to -2 if conduction of electrons is generated by hopping, whereas ballistic transport should appear in a different plot. Clearly, the experimental data shown in Fig. 2 fits Mizutani's theory whenever the unit cell of the crystal becomes large enough, in the vicinity of a crossover value $\beta_{\mathrm{Cc}} \approx 5$ (i.e. around 150 atoms per unit cell).

A complexity gap separates periodic CMAs from aperiodic ones, which is easily pointed out in the middle of Fig. 1: no crystalline compound was discovered so far in the region $9<\beta_{\mathrm{C}} \leq 40$. Why such a gap occurs and why nature prefers the quasicrystalline solution instead of growing crystals of increasingly larger unit cells is not 
understood so far from the point of view of the electronic structure. On the one hand, the Hume-Rothery interference and orbital hybridization are evoked in the frame of the nearly free electron (NFE for short) model to explain the stability of both crystalline and quasicrystalline states [16]. However, except for details of the shape of the Jones zone, they do not introduce any difference between the two options. On the other hand, ab initio computations can handle only periodic boxes and therefore are of no help regarding the actual origin of periodicity, or its absence. We show in the next section that localization induced by the breakdown of Bloch's theorem when the dimension of the unit cell increases above the crossover value also goes with the raise of an increasingly large number of $d$-like states located immediately below the Fermi energy. This mechanism fits on the one hand with the change of transport mechanism already pointed out, and on the other hand allows us arguing that the symmetry of $d$-states, henceforth of hopping, must be involved in the formation of the quasicrystal.

\section{Formation of $d$-states in the vicinity of the Fermi level}

The sub-band of $d$-states has received detailed investigation by SXES in Al-TM-TM' CMAs [17]. Essentially, $d$-states of $\mathrm{TM}=\mathrm{Cu}, \mathrm{Pd}, \mathrm{Ni}$ species lie few eV under the Fermi edge and interact with $3 s, d$, and $3 p$ states of Al which they split into bonding and antibonding states. The density of $\mathrm{Al} 3 s$ and $p$ states at the Fermi energy is low compared to that in fcc $\mathrm{Al}$ [18]. In contrast, $d$-states originating from $\mathrm{TM}^{\prime}=\mathrm{Fe}, \mathrm{Mn}$, Co element overlap the Fermi level and the position of their maximum intensity varies within a narrow range of 1 to $2 \mathrm{eV}$ around $E_{\mathrm{F}}$. By principle of the SXES technique, the relative weight of such $d$-states cannot be extracted from experimental data.

To overcome this difficulty, we shall study alloys that contain no transition metal, but only normal metals. A good choice is the $\mathrm{Al}-\mathrm{Mg}$ system, which contains the Samson $\beta-\mathrm{Al}_{3} \mathrm{Mg}_{2}$ phase with 1178 atoms per cubic unit cell (lattice parameter $a=2.8242 \mathrm{~nm}$ ) and its low temperature rhombohedral version (879 atoms) [19]. Both species, $\mathrm{Al}$ and $\mathrm{Mg}$, crystallize in the most simple way for a metal: $\mathrm{Al}$ is fcc (4 at./u.c.) and $\mathrm{Mg}$ is hcp (2 at./u.c.). They exhibit virtually zero $d$-states at the Fermi level [20]. For comparison purposes, we shall also study other phases from the Al-Mg system, but which contain a transition metal, like the $\mathrm{Mg}_{2} \mathrm{Zn}$ Laves phase (12 at./u.c.) and the AlMgZn Bergman phase (162 at./u.c.).

Part of the SXES data is shown in Fig. 3 for the $s, d$ sub-band, with the measurements collected at the Al edge in the left hand side of the figure, and the ones relative to the $\mathrm{Mg}$ edge in the other side. A sharp peak, marked by an arrow, develops 1 to $2 \mathrm{eV}$ under the Fermi level. Its width at half maximum is in the range of 1 to $2 \mathrm{eV}$ as well, and its position is weakly dependent upon the nature of

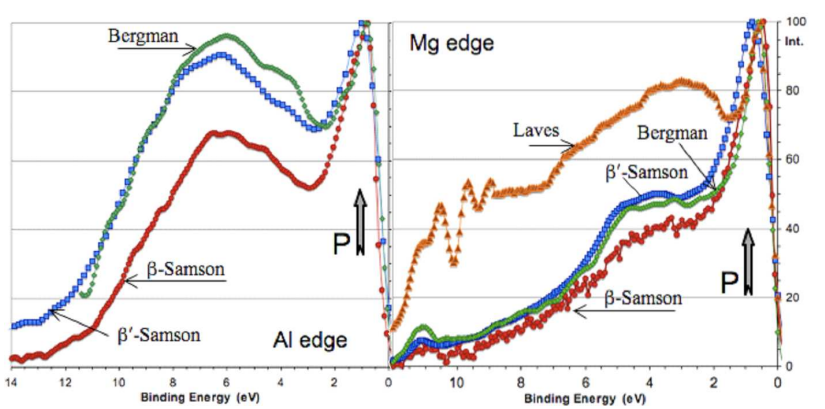

Fig. 3. SXES data at the $\mathrm{Al}$ edge (left) and $\mathrm{Mg}$ edge (right) gained for the $\mathrm{Al}-\mathrm{Mg}(-\mathrm{Zn})$ phases. Observe the narrow peak (marked by an arrow) that develops just under the Fermi energy taken as the origin of the binding energy scale.

the phase. In contrast, its intensity changes by a factor of about 4 when going from the Laves phase (of course, no data is available for the Al edge) to the Samson phase. Such a narrow peak characterizes localized states and is very similar to the peak found in the vicinity of $E_{\mathrm{F}}$ for the $d$-band in TM-containing CMAs [17]. Therefore, we shall refer to it as a peak due to " $d$-like" states.

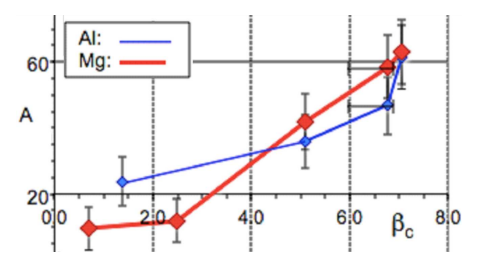

Fig. 4. Integrated intensity $A$ (in arbitrary units, after withdrawing the $\mathrm{Al} 3 s$ contribution underneath, extrapolated as a flat background) of the $d$-like peak pointed out in Fig. 3 as a function of $\beta_{\mathrm{C}}$. The red line is for $\mathrm{Mg}$ data and the blue one for Al data. Phases represented here are, from left to right, hcp $\mathrm{Mg}$ and fcc $\mathrm{Al}, \mathrm{Mg}_{2} \mathrm{Zn}$ Laves phase (no $\mathrm{Al}$ data), $\mathrm{Al}-\mathrm{Mg}-\mathrm{Zn}$ Bergman phase, $\beta^{\prime}-\mathrm{Al}_{3} \mathrm{Mg}_{2}$ and $\beta-\mathrm{Al}_{3} \mathrm{Mg}_{2}$.

Very surprising is the change of the area under the $d$-like peak when plotted as a function of the complexity index of the sample (Fig. 4). It increases with increase of complexity and therefore contributes more to the stability of the most complex phase than to the others. The increase is approximately linear with $\beta_{\mathrm{C}}$, i.e. the relative weight of $d$-like states compared to $s$-states increases exponentially with the number of atoms in the unit cell (extrapolation of this conclusion to the highest values of $\beta_{\mathrm{C}}$ is however not justified!). The origin of the $d$-like peak must therefore be found in the enhancement of localization that goes with the progressive loss of periodicity. Henceforth, we conclude at this stage that localization, or more appropriately self-organised criticality after Sect. 3 above, enforces the formation of $d$-like states placed on the binding energy scale $1-2 \mathrm{eV}$ below (and overlapping) the Fermi energy. 
In the following, we shall assume that the relative weight of $d$-like states near the Fermi energy originating in SOC is at least of the same order of magnitude, if not larger, in non-periodic samples compared to periodic ones with the highest values of $\beta_{\mathrm{C}}$. It is hidden under the $\mathrm{TM}^{\prime} d$ peak in TM-containing specimens from which it cannot be separated using SXES $\left(\mathrm{TM}^{\prime}=\mathrm{Fe}\right.$, $\mathrm{Mn}, \mathrm{Co})$. Such states are not extended in energy space like the other $s$ and $p$ states. Nevertheless, they participate in the transport mechanism by hopping if empty states are available and if thermal activation allows since they are located close to the Fermi energy. This way, they contribute to soften the pair potential in real space. The quasicrystalline state is remarkable in the sense that it represents a minimum of the contribution of $\mathrm{Al} 3 p$ and Al $3 s$ DOS to the free energy of the system and the most pronounced pseudogap [18]. This means that the energy barriers to overcome for hopping electrons at $E_{\mathrm{F}}$ are the highest when the symmetry of the lattice is icosahedral.

\section{Why are the atoms where they are?}

The question of the origin of missing translational symmetry in quasicrystals was already paused by few authors. Molecular dynamics simulations in a specifically designed double-well potential, that combines a classical Lennard-Jones part and a separated Gaussian valley, were able to prove that appropriate tuning of this type of potential stabilizes a decagonal arrangement of particles [21]. Later on, Lifshitz and Petrich could demonstrate, at least in the 2-dimensional case, that a fluid may exhibit a forbidden 12-fold pattern of the Faraday waves when driven by a linear combination of two frequencies [22]. Although their conclusion stressed that this model "is clearly not adequate for studying quasicrystals in the solid state", they emphasized that any physical system can be tuned to non-periodic if two competing wavelengths undergo a supercritical bifurcation as the one incorporated in their model.

Barkan et al. extended this work to the behaviour of isotropic charged particles [23]. They could show that a simple isotropic potential in the Fourier space, but with two minima the positions of which are the control parameters of the model, can stabilize a 12-fold array of particles. A key conclusion of these works is that the ratio between characteristic wavelengths must be taken in the vicinity of an irrational number, which stresses how sensitive to small variations of this ratio the system is $[22,23]$. We are therefore inclined to look upon a similar mechanism in CMAs that will produce competing interactions whose fine-tuning will eventually differentiate between crystals with a long period and quasicrystalline order.

Mizutani studied in numerical simulations the contribution of $d$-states to the stability of TM-based gamma brass phases having 52 at./u.c. [24]. He could conclude that a broadening of the gap between sub-bands takes place, due to the orthogonality of $s, p$, and $d$ wave functions, resulting in enhanced stability of the phase due to what he calls " $d$-states mediated Fs-Jz interactions". This phenomenon, which is unique to CMAs [24], induces a splitting between bonding states that are pushed towards higher binding energies, and antibonding states that are repelled above the Fermi energy. It has a spatial symmetry, expressed by the reciprocal lattice vector(s) that define the Fs- $\mathrm{Jz}$ interaction(s).

So now, why are the atoms where they are? At this stage, we may only speculate a scenario to explain the selection of the quasicrystal above the $9<\beta_{\mathrm{C}}<40$ formation gap. The first step is to check the enhancement of the density of $d$-like states near $E_{\mathrm{F}}$ as a function of the increasing size of the unit cell. This can be achieved nowadays up to few hundreds of atoms per unit cell. The fact that transport properties are rather similar in quasicrystals and in periodic CMAs of largest unit cell suggests that the number of $d$-like states saturates to some (unknown) value. Hence, the existence of an enhanced density of $d$-like states near $E_{\mathrm{F}}$, taken alone, will not make any substantial difference between periodic and aperiodic CMAs. Their role in the stability and transport mechanism must however become essential above the crossover value of $\beta_{\mathrm{C}}=\beta_{\mathrm{Cc}}$.

The next step will be to understand the dynamics of $d$-states and more importantly, its symmetry, in the conduction mechanism: the larger the respective weight of $d$-like states, the more reduced is conductivity, which implies that energy barriers arising from Fs- Jz interactions and orthogonality between wave functions are both key to the hopping mechanism. Nevertheless, Fs-Jz interactions of $s, p$ states on the one hand, of $d$-like states on the other in the presence of thermal activation of hopping (since quasicrystals form at high temperature) are able to install two competing wavelengths, via Friedel oscillations, which ultimately may explain why the periodic crystal becomes unstable compared to the quasicrystal above a certain proportion of $d$-like states, correlatively above a certain $\beta_{\mathrm{C}}$ threshold (which is approximately $\beta_{\mathrm{C}}=9$ in Al-based CMAs).

Third, a distortion of the lattice, like a Peierls transition, may take place when the Jones zone has near icosahedral symmetry in order to lower further the energy of the $d$-band. This mechanism would also contribute to suppress translation periodicity at high $\beta_{\mathrm{C}}$. The respective importance of such a mechanism and of phason entropy remains an open question.

\section{Conclusion}

This article was dedicated to an attempt to answer the most fundamental question still open in the physics of quasicrystals made of metals: why are the atoms where they are? We focused on a series of compounds based on aluminium, including icosahedral quasicrystals, but also many other simpler crystals. Observing that transport properties scale as a simple power law of the size of the unit cell, with slope equal to -1 , we concluded from this evidence of self-organized criticality that a drastic change 
of transport regime occurs when the number of atoms becomes larger than about 150 at./u.c. Simultaneously, we could remark that no periodic compound forms above some 10,000 at./u.c. and raise the question to know why nature prefers the quasiperiodic solution above this limit rather than to grow crystals with a unit cell of increasingly larger size. The formation of $d$-like states, i.e. energetically localized states, just under the Fermi level was pointed out in transition metal free compounds, indicating that the density of such states grows in proportion to the complexity of the unit cell and therefore contributes increasingly to stabilize the most complex lattice.

A speculative model was suggested in order to promote discussion with experts of the electronic structure and generate new research on the origin of quasiperiodic order in metallic alloys. It is based on: (i) the formation of $d$-states under the Fermi level that goes in proportion to the lattice complexity, (ii) the assumed occurrence of two competing wavelengths, one associated with the Fs-Jz interaction, the other with the hopping mechanism that transports electrons at finite temperature and (iii) possibly, a Peierls distortion that stabilizes the icosahedral lattice to the expense of the periodic ones of largest unit cell whenever $\beta_{\mathrm{C}}>8-10$. A toy model in 2 -dimension is presumably within reach of present computers and might be designed to test these ideas. The real 3D case most probably lies beyond the capabilities of today's computational physics. Whatever the value of this model, we insist on two points: (a) the participation of $d$-states in the selection mechanism of quasicrystals cannot be ignored, (b) numerical simulations in periodic boxes, assuming Bloch waves, cannot help understanding why quasicrystals form.

\section{Acknowledgments}

We are most grateful to H. Combeau (IJL, Nancy) for discussions about SOC, J. Dolinšek (JSI, Ljubljana) for the permission to use Ref. [8] prior to publication, and U. Mizutani (NISRI, Nagoya) for his guidance into his own work.

\section{References}

[1] Complex Metallic Alloys: Fundamentals and Applications, Eds. J.M. Dubois, E. Belin-Ferré, Wiley, Weinheim 2011.

[2] I. Mizutani, M. Inukai, H. Sato, E.S. Zijlstra, Chem. Soc. Rev. 41, 6799 (2012).

[3] G. Tramblyde Laissardière, D. Mayou, Phys. Rev. B 55, 2890 (1997).

[4] E. Belin-Ferré, J. Phys., Condens. Matter 14, R789 (2002).
[5] J.M. Dubois, in: Properties and Applications of Complex Intermetallics, Vol. 2, Ed. E. Belin-Ferré, World Scientific, Singapore 2008, p. 1.

[6] J.M. Dubois, Useful Quasicrystals, World Scientific, Singapore 2005.

[7] M. Quiquandon, Y. Calvayrac, A. Quivy, F. Faudot, D. Gratias, in: Quasicrystals, Preparation, Properties and Applications, Eds. J.M. Dubois, P.A. Thiel, A.P. Tsai, K. Urban, Vol. 553, Materials Research Society, Warrendale 1999, p. 95.

[8] M. Klanjšek, S. Jazbec, M. Feuerbacher, J. Dolinšek, Intermetallics 39, 50 (2013).

[9] C. Berger, in: Lectures on Quasicrystals, Eds. F. Hippert, D. Gratias, Les Editions de Physique, Les Ulis 1994, p. 463.

[10] E. Belin-Ferré, M. Klanjšek, Z. Jagličić, J. Dolinšek, J.M. Dubois, J. Phys., Condens. Matter 17, 6911 (2005).

[11] J. Dolinšek, T. Apihm, P. Jeglič, I. Smiljanić, A. Bilušić, Ž. Bihar, A. Smontara, Z. Jagličić, M. Heggen, M. Feuerbacher, Intermetallics 15, 1367 (2007).

[12] E. Belin-Ferré, J.M. Dubois, Philos. Mag. 88-13-15, 2163 (2008).

[13] P. Bak, How Nature Works: the Science of SelfOrganized Criticality, Copernicus Press, New York 1996.

[14] P. Bak, C. Tang, K. Wiesenfeld, Phys. Rev. A 38, 364 (1988).

[15] C. Sire, in Ref. [9], p. 505.

[16] U. Mizutani, Introduction to the Electron Theory of Metals, Cambridge University Press, Cambridge 2001, p. 509.

[17] E. Belin-Ferré, Z. Dankhazi, M.F. Fontaine, M.C. de Weerd, J.M. Dubois, Croat. Chem. Acta 83, 55 (2010).

[18] E. Belin-Ferré, V. Fournée, J.M. Dubois, J. Phys., Condens. Matter 12, 8159 (2000).

[19] W. Steurer, Z. Kristallogr. 222, 259 (2007).

[20] D.A. Papaconstantopoulos, Handbook of the Band Structure of Elemental Solids, Plenum Press, New York 1986.

[21] M. Engel, H.-R. Trebin, Phys. Rev. Lett. 98, 225505 (2007).

[22] R. Lifshitz, D.M. Petrich, Phys. Rev. Lett. 79, 1261 (1997).

[23] K. Barkan, H. Diamant, R. Lifshitz, Phys. Rev. B 83, 172201 (2011).

[24] U. Mizutani, Hume-Rothery Rules for Structurally Complex Alloy Phases, CRC Press, Taylor \&Francis, Boca Raton 2011. 